\title{
On the Conceptual Metaphor of Chinese Characters Yin and Yang in Yellow Emperor's Inner Canon
}

\author{
Huan Xu, Quansheng Tao \\ School of Foreign Languages, Anhui University of Science and Technology, Huainan 232000, Anhui, China
}

\begin{abstract}
In TCM culture, it is very common to see metaphors. We can apply what we have already known about the world to the biological phenomena and the regulation of diseases which we may not know a lot. Based on this kind of analogy, it forms many metaphorical concepts or conceptual metaphors. Yin and Yang, as a pair of Chinese characters with high frequency in TCM classics such as Yellow Emperor's Inner Canon, are a great example of the use of conceptual metaphor. In the past years, many researchers have devoted themselves to studying the meaning of these two Chinese characters, in particular, in the context of Yin-Yang theory. However, previous studies fail to do a systematic research on the conceptual metaphor of Yin and Yang. Therefore, this paper is concerned with the study of Yin and Yang from two different aspects-the meaning of Yin and Yang in Yellow Emperor's Inner Canon and the conceptual metaphor of Yin and Yang in the Yin-Yang theory. In the process of research, the author reads a number of documents and TCM classics concerning Yin and Yang. Finally, the author comes to the conclusion that the conceptual metaphor of Yin and Yang in TCM can be summarized to stand for the attributes of two opposite aspects of interrelated things or phenomena, and Yin and Yang possess four different kinds of relations among them. Besides, the formation, development and changes of all things result from the contradictory movement of Yin and Yang with unity of opposites. Or we can say that the four relations of Yin and Yang run through TCM when it comes to body structure, physiology, pathology and pulse manifestations. The research on the conceptual metaphor of Yin and Yang in Yellow Emperor's Inner Canon, in a sense, can help people better understand the conceptual metaphor in TCM language and figure out the meaning of Yin and Yang.
\end{abstract}

Keywords: Conceptual Metaphor, Yin and Yang, Yellow Emperor's Inner Canon.

\section{Introduction}

When With the development of China's economy, people are becoming more and more interested in various aspects of China's development, especially in terms of Traditional Chinese Medicine. However, many people still hold TCM in suspicion, arguing that TCM lacks scientific support. In fact, TCM is anything but unscientific. TCM is rooted in Chinese culture, and lots of its theories and treatments are closely related to Chinese philosophy. Philosophy is just one of the broad sciences. That's to say, it is inappropriate to reach the conclusion that TCM is unscientific. On the other hand, it also indicates the urgency of promoting the scientific nature of TCM culture. To this end, many researchers have been committed to the study of TCM language.

Yin and Yang, as a pair of Chinese characters with high frequency in TCM classics, are also attracting more and more researchers' attention. Many researchers have devoted themselves to studying the meaning of these two Chinese characters, in particular, in the context of Yin-Yang theory. However, previous studies fail to do a systematic research on the conceptual metaphor of Yin and Yang, Therefore, this paper is concerned with the study of Yin and Yang from two different aspects - the meaning of Yin and Yang in Yellow Emperor's Inner Canon and the conceptual metaphor of Yin and Yang in the Yin-Yang theory. In this regard, a systematic research on the meaning of Yin-Yang is necessary, which can help people better understand TCM.

\section{Literature Review}

The culture of TCM is based on its metaphorical nature. In this vein, TCM language has its own characteristics. In other words, conceptual metaphors are common in TCM language but we cannot separate them from Chinese culture.
Based on this point, today more and more scholars turn their efforts to the study of metaphors in TCM. Some scholars point out that a language is created by metaphorical model, and TCM language is no exception (Xie Jing and Jia Chunhua, 2011). According to Tu Jinli (Tu Jinli, 2008), metaphor phenomena in TCM can be divided into Yin-Yang metaphor, five elements metaphor, qi metaphor, natural metaphor, etc., which are based on different source domains in Yellow Emperor's Inner Canon. Rested upon Yin-Yang cognitive model, the theory of storing and releasing of zang-fu can be explained through mutual hiding between Yin and Yang, steady Yin and vexed Yang, and the like (Li Chenghua and Sun Huiming, 2016). In light of linguistics, the metaphorical model in some TCM classics is characterized by both systematization and consistency, both universality and empiricism, both variety and extensiveness. In addition, the metaphors in TCM represent a kind of dynamic metaphor (Shi Yingying, 2017).

However, other scholars tend to explain the concept of Yin and Yang in the theories of TCM. According to Qian Huinan (Qian Huinan, 2015), Yin and Yang mark a pair of opposite concepts in Yin-Yang theory. For example, up is Yang and down is Yin; heat is Yang and coldness is Yin. In fact, Yin and Yang can be summarized to stand for the attributes of two opposite and correlative things, reflecting the general feature of existence and changes of things in the world. In a world, Yin and Yang can be used to explain the origin, existence and evolution of the universe (Jing Hong, 2000).

On the other hand, many philosophical terms are also affected by the western philosophical concepts. Under the backdrop of western scientific system dominating almost every field, it is not uncommon to see that the original meaning of Yin and Yang is also confined to materialistic dialectics. In specific, Yin and Yang marks the appositive of contradictions or the 
unity of opposites (Hao Yu and He Juan, 2017). However, it is not fairly enough to explain the meaning of Yin and Yang.

Beyond the above, some scholars focus their study on the specific meaning of Yin and Yang from their application in TCM. For example, Meng Lingjun (Meng Lingjun, 1991) proposes that the back pertaining to Yang and the abdomen pertaining to Yin do not necessarily mean Yin and Yang refer to the front and back of the body respectively. In contrast, here Yin and Yang can refer to the upper part of the body and the lower part of the body separately. In terms of the theory of zang-fu organs, Yin and Yang can be used to divide zang-organs and fu-organs into different categories, such as liver pertaining to Yin while gallbladder to Yang (Zhang Xiaoxia and Zhang Canjia, 2002). As shown by differentiation of eight principles in TCM, Yin and Yang are applied to identify different diseases. For instance, the pathological symptoms manifested as chronic, deficiency-cold or hypo-active can be identified as Yin, while the pathological symptoms manifested as acute, hot and dry or hyperactive can be identified as Yang (Qiao Hongshuang, 2016). Additionally, Yin and Yang set up the foundation to distinguish different pulses according to their various manifestations (H M and G L, 2004). For example, the deep, slow and deficient pulse manifestations belong to Yin, while floating, multiple and sufficient pulse manifestations belong to Yang.

Although, the studies on the conceptual metaphor of Yin and Yang in TCM is common to find, but it is barely to find any systematic and comprehensive researches on the meaning of Yin and Yang. Furthermore, most of the available studies can hardly get rid of the shackle of philosophical concept of Yin and Yang. As a result, the author, combining the efforts of our predecessors, hopes to give more details about the meaning of Yin and Yang in TCM in a more systematic way by virtue of collecting and organizing sizable information. And in this paper, the author will focus on the conceptual metaphor of Yin and Yang from two aspects, namely, the meaning of Yin and Yang in Yellow Emperor's Inner Canon and the conceptual metaphor of Yin and Yang in the Yin-Yang theory.

\section{The Meaning of Yin and Yang in Yellow Emperor's Inner Canon}

In Yellow Emperor's Inner Canon, quite a number of chapters have mentioned Yin and Yang. Hence, we can see that Yin and Yang represent the code of TCM. Yin and Yang are indispensable parts of the secret of our life. No matter how complex our body is, Yin and Yang can figure it out. Also, no matter how marvellous our physiological mechanism is, Yin and Yang can figure it out. Based on the above facts, therefore, it will be not too hard for us to explain the pathology through Yin and Yang. Surprisingly, Yin and Yang can also be used to divide different pulses.

\subsection{Yin and Yang in Body Structure}

Yin and Yang in TCM are more focused on the two opposite attributes of the human and nature (Hao Yu and He Juan, 2017). As shown in Yellow Emperor's Inner Canon, the human body is an organic whole. The constituent parts of our body are both interrelated but also opposite. Therefore, different parts and changes of correlative human body parts can also be classified into Yin group and Yang group according to their different location and function (Fang Hui and Xiao Dangsheng, 2018).

Firstly, as for the body parts, the upper parts of the body pertain to Yang while the lower parts of the body pertain to Yin; the surface parts of our body pertain to Yang while the inside parts of our body pertain to Yin; the medial aspect of the limbs attributes to Yin while the lateral to Yang.

Secondly, in light of zang-fu organs, since five zang organs store essence without leaking, they pertain to Yin; while six fu organs, as digesting and transporting food and drink without storing essence, belong to Yang (Meng Lingjun, 1991). Besides, zang organs and fu organs can still be divided into Yin and Yang further. For example, the heart can be further divided into the heart-Yin and the heart-Yang; the stomach can be further into the stomach-Yin and the stomach-Yang.

Generally speaking, Yin and Yang can not only represent different parts of human body structure but also can summarize the inner nature of the body parts they represent.

\subsection{Yin and Yang in Physiology}

Yin and Yang have profound meanings in TCM and they can also be used to explain physiological function. As is known to all, the mystery of nature's development lies in that it is always in a state of dynamic balance, which is also the code of human's life activities. In TCM, the normal life activities of the human body result from the harmonious relation of the unity of opposites between Yin and Yang. As mentioned in Yellow Emperor's Inner Canon, only when Yin and Yang are in a state of balance and coordination can man be in good health. But when Yin and Yang become dissociated, we will end our lives for the loss of essence (“阴平阳秘, 精神乃绝; 阴阳离绝, 精气乃绝”).

When it comes to physiology, both Yin and Yang can be used to account for the relationship between substances and their functions. In this regard, the substances pertain to Yin while functions to Yang. Still not only such, substances can also be divided into Yin and Yang among themselves, so do their functions. As a rule, there are two kinds of opposite physiological performances. The first one such as down, astringent, restrained, etc. refers to Yin; the second such as up, out, vigorous, etc. refers to Yang. In order to illustrate this point, below are some particular instances.

Firstly, since we have mentioned that five zang organs belong to Yin while six fu organs belong to Yang, we can also know that five zang organs function as storing essence without leaking while six fu organs function as digesting and transporting food and drink without storing essence. This suggests that functions featuring storing essence without leaking stand for Yin; functions featuring digesting and transporting food and drink without storing essence stand for Yang.

Then going further, since five zang organs can be divided into Yin and Yang (Zhang Xiaoxia and Zhang Canjia, 2002), thus 
the functions of five zang organs can be divided to Yin and Yang too. Now, let's take the function of heart as an example. If the heart functions as nourishment and restriction, then we can call such a performance Yin. But if the heart functions as warming and promotion, then we can call this performance Yang.

From the above, we can perceive that Yin and Yang are not confined to divide the body parts. On the contrary, Yin and Yang are also available for stating the division of Yin and Yang in terms of physiology. And the relation of two types of functions can be characterized by mutual rooting and constraint. Under certain conditions, they may transform into the opposite side.

\subsection{Yin and Yang in Pathology}

The balance of Yin and Yang, to a large extent, reflects a person's physical condition (Bellavite and Semizzi, 1998). When this kind of balance is disrupted, different pathological manifestations will follow, such as excess of Yin or Yang, deficiency of Yin or Yang, mutual loss of Yin and Yang, etc. (H M and G J, 2004).

Firstly, excess of Yin or Yang, as the name suggests, indicates Yin excess or Yang excess. On this condition, Yang excess leads to heat syndrome while Yin excess causes cold syndrome (阳胜则热, 阴胜则寒). Yang, is characterized by hot, hyperactive, acute, and so forth, and thus when the pathological state is manifested as hot, hyperactive or dry, sometimes even following rapid pulse, etc., then we can call such a state the excess of Yang. Tellingly, excess of Yin is a kind of opposite pathological state compared with excess of Yang. Its pathological manifestations are often described as follows - cold, chronic, hypo-active, etc. Since Yin flourishes while Yang declines, the patient loves to be warmed, and sometimes together with stomachache, powerless pulse, and so on. So the next time when a man feel chill, tasteless but not thirsty or more of the above syndromes, we may make a judgment that this man suffers from the excess of Yin.

Secondly, deficiency of Yin or Yang is a kind of pathological change that caused by the depletion of Yin fluid or Yang qi. As talked about in Yellow Emperor's Inner Canon, Yang deficiency leads to cold outside while Yin deficiency causes heat inside (阳虚则外寒, 阴虚则内热). In general terms, Yang qi can be used to express this kind of qi in human body whose movement can be highlighted as up, hyperactive, clear, and so forth., and this kind of stuff have an important role in a man's metabolism and his normal physiological functions (Piao Yuanlin, Yin Dehai, 2018). Yang deficiency, in fact, refers to the deficiency of Yang qi. Once Yang qi is deficient, human body will appear a series of pathological changes like the zang-fu organs becoming weakened, the blood movement slower, the face paler, and the limbs colder, and the like. So when it comes to Yang deficiency, we mean the pathological manifestations are similar to the above conditions. On the other hand, Yin fluid refers to a kind of fluid that is rich in nourishment in a general sense, and often fluid is thick and turbid so it belongs to Yin. In terms of pathology, Yin deficiency can also be interpreted as the depletion of Yin fluid. In TCM, Yin fluid includes blood, essence, etc. If Yin fluid is deficient, by comparison, Yang qi will be more active. In this case, the pathological manifestations are more prone to "Yang", which means fever in chest palms-soles, night sweat, dry throat and tongue, weak pulse, etc. In part, Yin deficiency is equivalent to the state that body functions are hyperactive.

Thirdly, Both Yin and Yang will get declined when Yin and Yang lose balance. That's to say, if Yin get declined, the development of disease will have a negative effect on Yang and Yang will also get decline; and vice versa. If clinical manifestations are shown as spermatorrhea, night sweat, fear of cold, and so on., then we can call it Yin deficiency affecting Yang; if clinical manifestations are shown as edema, agitation, thirst, and so forth, then we can call the state Yang deficiency affecting Yin (Li Fei and Zhai Shuangqing, 2010).

From the above, it is safe to conclude that once the relation of waxing and waning of Yin-Yang are abnormal, diseases will follow. In some cases, the diseases may even transform from Yin symptoms into Yang symptoms or vice versa, which is known as the transformation between Yin and Yang. Considering this point, we can see that Yin and Yang can be used to evaluate a basic core aspect of individual's pathological manifestations.

\subsection{Yin and Yang in Terms of Pulse Manifestation}

Yin and Yang are not only the foundation of differentiation of diseases, and they also set up the foundation for distinguishing different pulses (Qian Huinan, 2015). Many TCM classics also share the same opinion.

Firstly, Plain Questions (难经) has already clearly pointed out that one who are good at make a diagnosis, will distinguish between Yin and Yang through inspection and pulse-taking and palpation (“善诊者, 察色按脉, 先别阴阳”). From this point, we can understand that Yin and Yang are key to distinguishing and classifying different pulse based on their pulse manifestations. In this regard, Yin and Yang capable of recognizing different kinds of pulses, are largely based on the fact that different pulse manifestations often share some similar features with what Yin and Yang represent. In particular, these different pulse manifestations feature two opposite attributes.

Secondly, as displayed in Miraculous Pivot (灵枢), if the pulse manifestations present the following characteristics, such as deep, slow and deficient pulse, etc., then we can call such kind of pulse Yin pulse. Furthermore, if the pulse manifestations are shown as floating, multiple, sufficient, and the like, then this kind of pulse can be defined as Yang pulse (Huang Dan and Wu Chengyu, 2010).

Thereby, the classification of Pulse manifestation between Yin and Yang also reflect the relation of opposition and mutual rooting between Yin and Yang.

\section{The Conceptual Metaphor of Yin and Yang in the Yin-Yang Theory}

As mentioned in the previous session, it is clear to see that Yin and Yang in Yellow Emperor's Inner Canon have both various and concrete meaning. According to their practical 
application like body structure, physiology, pathology and pulse manifestations, then the conceptual metaphor of Yin and Yang in the Yin-Yang theory will be much easier for us to understand. In Yin-Yang theory, we can understand four different kinds of relations between Yin and Yang, such as opposition of Yin-Yang, mutual rooting of Yin-Yang, waxing and waning of Yin-Yang, and transformation between Yin and Yang. These four different relations are evidently not set casually. Instead, the four relations between Yin and Yang are established on the basis of observing the law of nature.

Originally, Yin represents the dark side of a slope while Yang represents the bright side of a slope (Kuang Jijia, 2018). Gradually, Yin and Yang have been summarized to stand for the attributes of two opposite aspects of interrelated things or phenomena in nature. Specifically, they can not only be used to represent two opposite attributes such as light and bright, heat and coldness, but also can be used to stand for two types of opposite movement tendency or state of motion such as static and dynamic, up and down. Consequently, TCM, as a part of natural science, can also apply the four different relations between Yin and Yang to the origin and nature of our lives. And the key to making a judgment on the attributes of Yin or Yang is up to its nature, position and tendency and so on. If we can understand what Yin and Yang represents in the four relations of nature, then it will be much easier for us to find the meaning of Yin and Yang in TCM.

\subsection{Conceptual Metaphor of Opposition of Yin-Yang}

Having talked about the basic meaning of Yin and Yang, we can understand that Yin and Yang represent a pair of opposite meaning. Be it the concrete things of the nature like water, fire, and so on., or some environmental changes in nature like sunrise, sunset, and so forth, the inclination of unity of opposites is quite common ( $\mathrm{Li} \mathrm{Jia}$ and Wang Dongzhou, 2011), which means that Yin and Yang can explain everything as long as they are possessed of opposite characteristic. In addition, we have already discussed the hidden meaning of Yin and Yang in light of body parts, physiology, pathology and pulse manifestation in the previous section. Next, we will see what Yin and Yang symbolize in the Yin-Yang theory. Firstly, opposition of Yin-Yang indicates that everything or phenomena in this world include two opposite aspects of Yin and Yang, like the sky and the earth, up and down, dynamic and static, and the like. In this context, the sky is Yang while the earth is Yin; up is Yang while down is Yin; dynamic is Yang while static is Yin (Zang Min and Liu Lei, 2018). This kind of contradiction is very common in this world, and Yin and Yang are the very best choice to explain it. As a matter of fact, opposition of Yin and Yang will lead to restriction and struggle between each other. Only when such kind of restriction and struggle comes to unity, then dynamic balance can be achieved.

\subsection{Conceptual Metaphor of Opposition of Yin-Yang in the Yin-Yang Theory}

We human being, as a kind of thinking animal, are adept in applying what we have observed to ourselves. Since the nature itself is filled with contradiction, then mankind living harmony with the nature will also be full of contradiction. Dealing with human body, the main contradiction of life phenomenon lies in the motive power of life development, which is throughout the course of our life. More specifically, the main contradiction refers to the struggle between living substances and vital functions. When comes to Yin and Yang, we can say that living substances can be represented by Yin while vital functions can be represented by Yang. According to Yellow Emperor's Inner Canon, our life is supported by the process of qi transformation. Since qi is always moving, we can call it Yang qi. While fluid is a kind of very important living substance, so we can name it Yin fluid. As a result, the essence of qi transformation marks the opposition of Yin fluid and Yang qi. Like in nature, only when Yin and Yang achieve unity in the process of restriction and struggle can the normal life activities be maintained. In general, opposition of Yin-Yang can be used to explain the two opposite sides of objects or phenomena and the relationship of mutual restriction.

\subsection{Conceptual Metaphor of Mutual Rooting of Yin-Yang}

Secondly, Yin and Yang are interdependent on one another for existence and cannot be cut apart (Liu Hongcai, 2017). For example, up is Yang and down is Yin; heat is Yang and coldness is Yin, daytime is Yang and night is Yin. However, there will be no down if up does not exist. And there will be no heat if coldness does not exist. Similarly, there will be no daytime if night does not exist. That's to say, what Yin represents and what Yang represents are not only in relation to mutual exclusion but also interdependence. It indeed makes sense because the original meaning of Yin and Yang is a very good example of this point - there will be no facing the sun when backing the sun is excluded.

\subsection{Conceptual Metaphor of Mutual Rooting of Yin-Yang in the Yin-Yang Theory}

In Plain Questions (素问), Yang qi is rooted in Yin, and vice versa; Yang cannot be produced when Yin does not exist, and Yin cannot be transformed when Yang does not exist (“阳气 根于阴, 阴气根于阳. 无阴则阳无以生, 无阳则阴无以化”). In this respect, mutual rooting of Yin and Yang is the essential factor that how things will change or develop. For instance, it is universally known that physiological activities are in close relation with living substances and vital functions. As mentioned before, we can see that different living substances, different vital functions or between living substances and vital functions are in relation of mutual rooting of Yin and Yang. Living substances belong to Yin while vital functions belong to Yang. Furthermore, Living substances play an essential part in vital functions while vital functions are the reflection of living substances. That's to say, when zang-fu organs function well, then nutrient substances can be produced. On the other hand, if our body has enough nutrient substances, then the normal functions zang-fu organs will be guaranteed. For instance, there is a saying that qi serves as the commander of blood and blood does as mother of qi (“气为血之帅, 血为 气之母”), which shows that Yin and Yang are mutually rooted. In addition, once the relationship of mutual rooting of Yin and Yang is destroyed, then diseases will come. In light of pathology, any loss of Yang qi will lead to the loss of Yin fluid, and vice versa. In order to maintain physical fitness, we cannot separate Yin and Yang from each other. 


\subsection{Conceptual Metaphor of Waxing and Waning of Yin-Yang in the Yin-Yang Theory}

Thirdly, the relation between Yin and Yang is not absolutely unchanged. On the contrary, Yin and Yang are always in the process of dynamic change. That means, waxing and waning follow the whole process (Jin Guangliang and Jia Hongbin, 1999). Under such circumstances, waxing and waning are absolute while dynamic balance is vis-a-vis. Usually, the process of waxing and waning of Yin - Yang can be divided into two types, namely the waxing and waning of either Yin or Yang and the waxing and waning of both Yin and Yang. For instance, physical function is booming in the daytime but weakening in the night. Excitatory state represents Yang while depressed state represents Yin. From excitatory state to depressed state, Yang wanes while Yin waxes and vice versa. In this regard, we can also call it Yang wanes while Yin waxes. However, there is always a limit for anything. Once we cross the critical point, the nature of things will also get changed. And that means the process of waxing and waning of Yin-Yang must maintained a balanced state. Otherwise, things will lose control.

\subsection{Conceptual Metaphor of Waxing and Waning of Yin-Yang in}

We human being are sensitive to the change of our own body. For example, one will feel weak and inactive when in a state of hunger. The reason for it is that Yin essence is not sufficient to support the production of Yang. Accordingly, Yang will also become less and less. In this case, we can view such kind of change as both Yin and Yang wane. In Plain Questions (素 问), we can also find a similar saying that Yin grows while Yang is generating and Yin conceals while Yang is restrained (“阳生阴长, 阳杀阴藏”). It thus appears that once the relationship of waxing and waning is over the limit of physiological ability, then our body will respond to this kind of change. What's more, it will harvest sickness fruits. But when one takes nutrition in time, then different from before, one will feel much better and have more strength. That's because nutrient substances which are also viewed as Yin, are transformed into energy that can be named Yang. As a result, both Yin and Yang waxes. To the end, Yin and Yang come to balance. Broadly speaking, the process of Waxing and waning of Yin and Yang is common to see both in nature and in human body, and it results from opposition of Yin-Yang and mutual rooting of Yin-Yang. But one thing we need to pay attention is that only when waxing and waning of Yin-Yang is in a state of balance can be a normal phenomenon, otherwise, it is abnormal and the nature of things will also get changed. Anyway, case by case principle marks the truth. What we need to do is to find out the of Yin and Yang under different contexts.

\subsection{Conceptual Metaphor of Transformation between Yin and Yang}

Lastly, Yin and Yang can transform into each other under given conditions. That's to say, things will develop in the opposite direction when in a phase of extremity-Yin can turn to Yang and Yang can turn to Yin (Ding Yu, 2012). We can also find the answer in an old Chinese saying - too much water drowned the miller (物极必反). When we say things or phenomena belong to Yin or Yang, we mean that the features of Yin or Yang hold a dominate position concerning a thing or phenomenon (Zhang Dengben, 2009). To put it in another way, we can never define the nature of things or phenomena by means of Yin and Yang in an absolute way. For instance, it is recorded in Miraculous Pivot (灵枢) that too much Yin will lead to Yang while too much Yang will lead to Yin; Yin dominates coldness while Yang dominates heat (“重阴必阳, 重阳必阴, 故阴主寒, 阳主热”) (Zhong Shu and Fang Ming, 2014). This indicates that the things or phenomena featuring coldness can be regarded as Yin while featuring heat can be regarded as Yang. When coldness prevails, then heat will also become colder, and vice versa. Actually, the process of transformation between Yin and Yang also includes waxing and waning of Yin-Yang. Thus this kind of change tells us that waxing and Waning of Yin-Yang is the premise of transformation between Yin-Yang and transformation between Yin and Yang is the result of waxing and waning of Yin-yang.

\subsection{Conceptual Metaphor of Transformation between Yin and Yang in the Yin-Yang Theory}

Likewise, transformation between Yin and Yang is not hard to find in our human body. In light of our life activities, living substances and vital functions can also transform into each other in the process of metabolism. This represents normal change between Yin and Yang. However, in terms of pathology, transformation between Yin and Yang is grouped into different types, like Yin symptoms and Yang symptoms, exterior symptoms and interior symptoms, cold symptoms and heat symptoms, etc. For instance, a patient suffering from Yang symptoms, which is characterized as ardent fever, agitated and so on, will turn to Yin symptoms that feature pale face and cold limbs when in the phase of severe illness. In general, transformation between Yin and Yang marks the basic law how things will change. Once met certain requirements, things will turn to its opposite side.

\section{Conclusion}

Our human is a part of nature. And if we can have a better understanding on the secret of nature, then we will know ourselves much better too. What nature can teach us is much more than what we have known, and that's why the author attempts to get inspiration from nature in order to figure out the conceptual metaphor of Chinese characters Yin and Yang in Yellow Emperor's Inner Canon. In their original meaning, Yin represents the side that back the sun and Yang represents the side that toward the sun respectively. That also means the nature is full of unity of opposites. As a part of nature, it is not difficult to say that we human beings are filled with unity of opposites as well. And Yin and Yang are the best representatives to show the traits. Hence, the author takes this as a start to help people to better understand the meaning of Yin and Yang in Yellow Emperor's Inner Canon.

In this paper, the author, based on the information she has learned and collected, mainly focus on two parts. The first one is about the meaning of Yin and Yang in Yellow Emperor's Inner Canon. In this part, the author talks about the specific meaning of Yin and Yang from four aspects, namely, body 
structure, physiology, pathology and pulse manifestations. And the second one, based on the different and concrete meaning of Yin and Yang in the first part, is concerned with the conceptual metaphor of Yin and Yang in the Yin-Yang theory, including opposition of Yin-Yang, mutual rooting of Yin Yang, waxing and waning of Yin-Yang and transformation between Yin and Yang. Finally, the author comes to the conclusion that the conceptual metaphor of Yin and Yang in TCM can be summarized to stand for the attributes of two opposite aspects of interrelated things or phenomena, and they include four different kinds of relations. Besides, the formation, development and changes of all things result from the contradictory movement of Yin and Yang with unity of opposites. Or we can say that the four relations of Yin and Yang run through TCM when it comes to body structure, physiology, pathology and pulse manifestations.

On the other hand, although the research focus and the research methods are valid, it still has room for improvement given the author's current academic level. By great efforts, the author hopes that the research on the conceptual metaphor of Yin and Yang in Yellow Emperor's Inner Canon, in a sense, can help people better understand the conceptual metaphor in TCM language and figure out the meaning of Yin and Yang. And for the acceleration of going abroad, it indeed needs great efforts for us to demonstrate the validity and effectiveness of Traditional Chinese Medicine. Therefore, it is our duty to be familiar with the knowledge of TCM, and the study on the Conceptual metaphor of Chinese characters Yin and Yang is just a small part of TCM. If so, everybody can do a fair share to the publicity of TCM through what they have learned.

\section{References}

[1] Xie Jing, Jia Chunhua. Types and Functions of Metaphorical Language in Yellow Emperor's Inner Canon. Journal of Traditional Chinese Medicine, 2011, 39 (01): 1-4.

[2] Tu Jinli. A cognitive study of TCM Metaphor in Yellow Emperor's Inner Canon. Tianjin: Master's Dissertation of Nankai University, 2008: 24-53.

[3] Li Chenghua, Sun Huiming, Zhang Qingxiang. Theoretical Interpretation of Zang-Fu organs and Diarrhea in Inner Canon Based on Metaphorical Cognition of Yin and Yang. Journal of Changchun University of Traditional Chinese Medicine, 2016, 32(6).

[4] Shi Yingying. On the Metaphorical Model and Its Characteristics in the Language of Traditional Chinese Medicine Classics. Cultural and Educational Information, 2017 (22): 14-15.

[5] Qian Huinan. Analysis of Yin Yang Pulse Method in Nanjing. Journal of Anhui University of Traditional Chinese Medicine, 2015, 34(5): 1-3.

[6] Jing Hong. Yin Yang and Five Elements Thought and Yellow Emperor's Inner Canon. Study on Zhouyi, 2000 (03): 86-91.

[7] Hao Yu, He Juan. Reflections on the Connotation of Yin and Yang in TCM. Journal of Beijing University of Traditional Chinese Medicine, 2017, 40(12): 973-977.

[8] Meng Lingjun. Discussion on the Annotation of "Back Is Yang While Abdomen is Yin". Journal of Shandong
University of Traditional Chinese Medicine, 1991 (03): 45-47.

[9] Zhang Xiaoxia, Zhang Canjia. On the Yin Yang Attribute of Zang-Fu organs and Its Genetic Principle. Journal of Shandong University of Traditional Chinese Medicine, 2002 (06): 450-453.

[10] Qiao Hongshuang. Experience of Yin Yang Syndrome Differentiation after Reading the Classic. Inner Mongolia Traditional Chinese Medicine, 2016, 35(17): 67.

[11] Langevin H M, Badger G J, Povolny B K, et al. Yin scores and Yang scores: a new method for quantitative diagnostic evaluation in traditional Chinese medicine research. The Journal of Alternative \& Complementary Medicine, 2004, 10(2): 389-395.

[12] Fang Hui, Xiao Dangsheng. Probing the Definition of Yin and Yang in Our Body. World Journal of Integrated Traditional and Western Medicine, 2018, 4 (01): 25-30.

[13] P. Bellavite, M. Semizzi, S. Lussignoli, G. Andrioli, U. Bartocci. A computer model of the 'five elements' theory of traditional Chinese medicine. Complementary Therapies in Medicine, 1998, 6 (3).

[14] Piao Yuanlin, Yin Dehai. Mechanism underlying treatment of diabetic kidney disease using Traditional Chinese Medicine based on theory of Yin and Yang balance. Journal of Traditional Chinese Medicine, 2018, $38(5)$.

[15] Li Fei, Zhai Shuangqing, Mei Yuezhang. Differentiation of "Eight Principles" in Yellow Emperor's Inner Canon. Journal of Beijing University of Traditional Chinese Medicine, 2010, 33 (11): 737-738.

[16] Huang Dan, Wu Chengyu. Application of Yin and Yang in Pulse Diagnosis. Jilin Traditional Chinese Medicine, 2010, 30(01): 6-7.

[17] Xie Hua. Yellow Emperor's Inner Canon: Interpretation and Translation on Vernacular. Traditional Chinese Medicine Classics Press, Beijing, China. 2000: 300.

[18] Li Jia, Wang Dongzhou, Lu Yanhong. The Harmonious Thoughts Research of Yin-yang and Five Elements Based on the Body. Energy Procedure, 2011, 13.

[19] Zang Min, Liu Lei, Bao Suzhen, et al. On the Development of Traditional Chinese Medicine Theory Based on the Application of Yin-yang Theory in Yellow Emperor's Inner Canon. Chinese Journal of Traditional Chinese Medicine, 2018, 33 (7): 2754-2757.

[20] Liu Hongcai. Yin Yang theory As Excellent Traditional Chinese Medical Culture. Research on Comprehensive Practical Activities, 2017 (6): 53-54.

[21] Jiang Guangliang, Jia Hongbing, Ding Shiqin. Discussion on the Theory of Yin and Yang Fluctuation in Inner Canon. Journal of Anhui University of Traditional Chinese Medicine, 1999 (01): 8-10.

[22] Ding Yu, Yin and Yang in TCM. People's Military Medical Publishing House, Beijing, China. 2012(2): 42-48.

[23] Zhang Dengben. Study on Yin Yang theory in Yellow Emperor's Inner Canon. Journal of Shanxi College of Traditional Chinese Medicine, 2009, 10 (02):2-8.

[24] Zhongshu Zhu, Fangming Liu. Discussion about "Jie-jie" theory in Lingshu (Miraculous Pivot). Chinese Acupuncture and Moxibustion, 2014, 34(11): 1087-8. 


\section{Author Profile}

Huan Xu received the B.A. degrees in Hubei University of Traditional Chinese Medicine in 2019. During 2020-2022, she will further study in Anhui University of Science and Technology to get a M.A. Degree.

Quansheng Tao professor, master tutor, School of Foreign Languages, Anhui University of Science and Technology. Research direction: foreign language teaching and research. 